\title{
Toward Improved Time Domain Stability and Passivity for Full-Wave PEEC Models
}

\author{
J. $\operatorname{Ekman}^{(1)}$, G. Antonini( ${ }^{(2)}$, A. E. Ruehli( ${ }^{(3)}$ \\ (1) EISLAB, Department of Computer Science \& Electrical Engineering \\ Luleå University of Technology, 97187 Luleå, Sweden \\ (2) UAq EMC Laboratory, Department of Electrical Engineering, University of L'Aquila \\ Poggio di Roio, 67040 AQ, Italy \\ (3) T. J. Watson Research Center, IBM Research Division \\ Yorktown Heights, NY 10598, USA
}

\begin{abstract}
It is well known that time domain integral equation techniques may suffer from stability problems and frequency domain models may provide non-passive results. A main source of these issues is the delay of the coupled elements. In the classical Partial Element Equivalent Circuit (PEEC) method, a single delay was used for each couple of partial element which results in a delay differential equation with reduced stability and accuracy. In this paper, we consider multiple delay coefficients which can be used for both the time and frequency domain. Also, filters are introduced which remove unwanted eigenvalues or resonances in the partial element couplings. This can substantially improve the response of the frequency domain and the time domain models. Stability improvements also means passivity improvements.
\end{abstract}

\section{INTRODUCTION}

The size of the electromagnetic problems which can be solved in today's solvers is relatively modest in comparison to the complexity of the electronic systems which have to be designed. An effort is made by the electromagnetic research community to extend the size of the problems which can be solved.

The Partial Element Equivalent Circuit (PEEC) method [1], [2] has shown to be particularly suitable for the solution of EMC and electrical interconnect and package (EIP) problems in combination with SPICE type circuit models since the entire problem is solved in the circuit domain. This approach also facilitates the enhancement of the PEEC model itself with conventional circuit elements. A key feature of the PEEC approach is that the same circuit models can be constructed for both the time and the frequency domain. In the classical implementation of the PEEC method, coarse approximation of the delays in the resultant delay differential equation (DDE) were used which impact both stability and accuracy. The time domain stability of integral equation based techniques has been a research topic for many years [3] since they result in a fast solution approach. Stability improvements also imply improvements in passivity since stability is a necessary condition for passivity.
In this paper we consider the stability and accuracy enhancement of the PEEC method by presenting an efficient filtering method in conjunction with a subdivision approach to allow the proper modeling of the amplitude and phase of the partial elements. The filter helps to remove unwanted resonance in the frequency dependent partial element coupling above the extended frequency range. Results for the filtering scheme are provided in the frequency domain which directly relates to model stability in the time domain.

\section{Partial Element Evaluation}

In the frequency domain, the delays are incorporated by multiplication of the static partial elements, (1) for partial inductances, by the exponential $e^{-s \tau}$ to account for the phase shift or delay in the electromagnetic field couplings which are of the form

$$
L_{p, \alpha \beta}^{S}(s)=\frac{\mu_{0}}{4 \pi a_{\alpha} a_{\beta}} \int_{v_{\alpha}} \int_{v_{\beta}} \frac{1}{\boldsymbol{r}_{\alpha}-\boldsymbol{r}_{\beta} \mid} \boldsymbol{u}_{\alpha} \cdot \boldsymbol{u}_{\beta} d v_{\alpha} d v_{\beta}
$$

In (1), $a_{\alpha, \beta}$ and $v_{\alpha, \beta}$ represent the cross section and volume of volume cells $\alpha$ and $\beta$, respectively, and $\boldsymbol{u}_{\alpha, \beta}$ their unit vectors. This is an approximation of the actual discrete coupling effect defined as

$$
L_{p, \alpha \beta}(s)=\frac{\mu_{0}}{4 \pi a_{\alpha} a_{\beta}} \int_{v_{\alpha}} \int_{v_{\beta}} \frac{e^{-s \tau}}{\boldsymbol{r}_{\alpha}-\boldsymbol{r}_{\beta} \mid} \boldsymbol{u}_{\alpha} \cdot \boldsymbol{u}_{\beta} d v_{\alpha} d v_{\beta}
$$

where $\tau=\left|\boldsymbol{r}_{\alpha}-\boldsymbol{r}_{\beta}\right| / c_{0}$ and $c_{0}$ is the speed of light in vacuum.

\section{A. Frequency dependent partial elements}

Recently much attention has been devoted to the more accurate computation of frequency dependent partial element [4], as shown in (2) for partial inductances. It was observed that the model stability substantially depends on these issues [5]. Figure 1 gives an example of the frequency dependence of the amplitude of the partial element mutual 


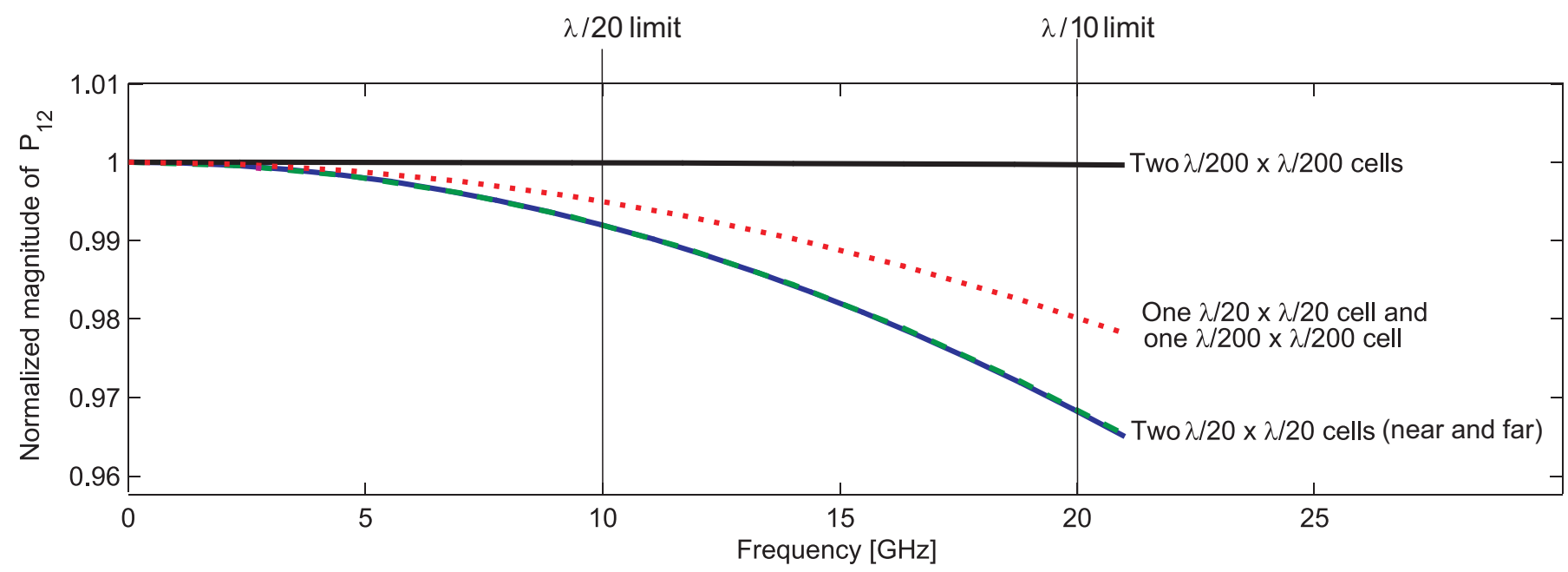

Fig. 2. The impact of damping in the active frequency range for a few different test cases.

coupling coefficients for the scalar potential which is of the same form as (1) and (2).

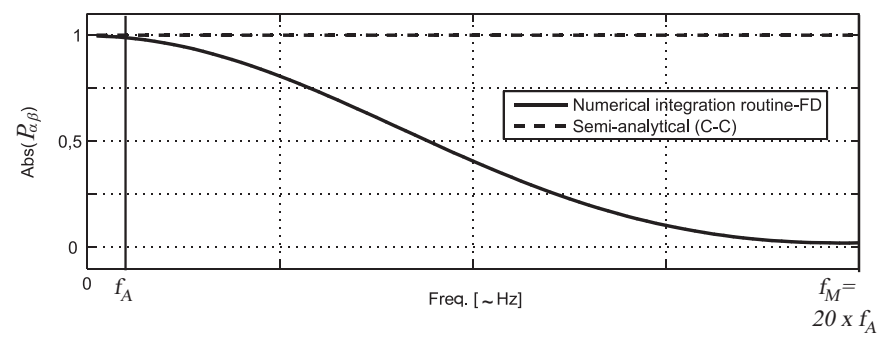

Fig. 1. Impact of delay on the magnitude of a coefficient of potential coupling for two different computational routines - one taking into account the frequency dependence (FD) and one using the classical center-to-center approximation (C-C).

It is obvious that the more accurate solution of the phase factor in the integrals drastically impacts the high frequency damping. This damping is not present in the classical PEEC delay approximation and it is obviously absent in the quasistatic model since delays are not included. The importance of the delay on the partial element was shown in recent work [6], [7] by constructing a macromodel for the partial elements which may be time consuming.

\section{B. Subdivisions}

Here we introduce an approach for the partial element coupling which is summarized as follows:

- The damping in the coupling is present in the active frequency range, $\left(f \leq f_{A}\right)$ where $f_{A}$ is determined by the meshing or the $\lambda_{\min } / 20$-limit. $f_{A}$ is the highest frequency of interest from a solution point of view. The damping in the active frequency range is clearly shown in
Fig. 2 for a few cases of mutual coefficients of potential coupling where $f_{A}$ is chosen to be $10 \mathrm{GHz}$.

- The damping issue for near and far spaced cells is similar, as expected from the phase behavior, provided that the cell size is the same. Figure 2 shows the result for both near and far coupling for two $\lambda / 20 \times \lambda / 20$ cells $\left(1.5 \times 1.5 \mathrm{~mm}\right.$ at $\left.f_{A}=10 \mathrm{GHz}\right)$. We normalized the magnitude to the same value to show the frequency behavior of the damping. Obviously, cells smaller than the conventional $\lambda / 20$ size will exhibit less damping. This is also evident from Fig. 2.

The accurate computation of the frequency dependent double or triple integrals, like (2), can be extremely time consuming if more efficient techniques are not applied. This is also the case for the quasi-static integrals. Hence, many techniques have been studied in order to speed up their computation e.g., [8]. One indirect solution to the integral problem was presented in [9] where it was suggested that the integrals be subdivided with a single delay associated with each subcell for the computation of the partial elements to improve the extended frequency range behavior. This technique also captures the frequency dependent behavior in the partial elements in an effective manner using a local subdivisions of the volumes and surfaces. For an efficient approach only few a subdivisions are used. The local volumes and surfaces can be subdivided into 9,12 , or a maximum of 16 local subdivisions. In [4] it was show that the degree of subdivision impacts the behavior in the extended frequency range for the partial elements.

\section{Very high-frequency response}

It is important to understand that reasonably good accuracy is required for frequencies above the active range limited by 
$f_{A}$. Experimentally, we pick a range up to $f_{M}$ which we call the maximum frequency of interest and $f_{M}=10$ to $20 f_{A}$. For this reason, we observe the behavior of the coefficients for frequencies much higher than $f_{A}$. Clearly, the damping behavior is of importance in the active range $f<f_{A}$ and also in the extended range $f_{A}<f<f_{M}$. Unfortunately, resonances appear even above $f_{M}$ which are due to a poor representation of the phase. This is shown in Fig. 3 for the case of 9, 16, and 25 local sub-cells. Importantly, these resonances are also present for accurate, time consuming techniques like Gaussian integration as shown in [4]. Figure 3 shows that increasing the number of sub-surfaces may move the resonances to higher frequencies. However, the impact of this on the stability or accuracy cannot be predicted in isolation of the entire PEEC model. Interferences will occur among the cells for problems with multiple cells leading to other instabilities.

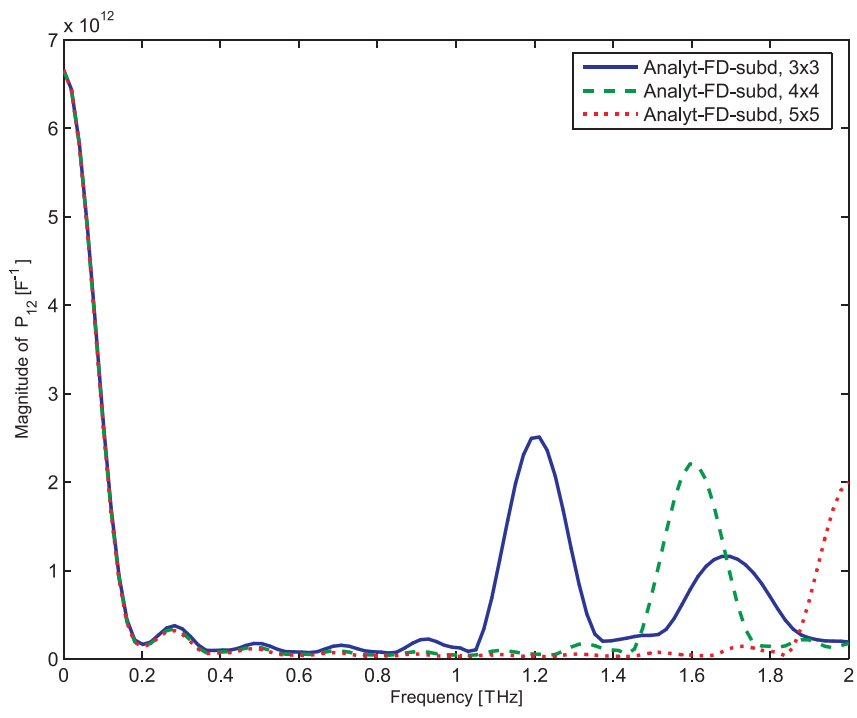

Fig. 3. Impact of the number of subdivisions on the magnitude of mutual coefficient of potential. $f_{A}=10 \mathrm{GHz}$ and $f_{M}=200 \mathrm{GHz}$.

\section{HIGH FREQUENCY FILTERING}

Another key element used in the stabilization is the filtering of the resonance above $f_{M}$ since increasing the number of subdivisions is not an efficient approach. The augmentation of the subdivided partial elements technique, from the previous section, with built-in filters, represents an effective overall stabilization approach. Elements of filtering are considered in [9] for partial inductances and in general in [10]. It is evident from the resonance behavior, as can be observed in Fig. 3, that arbitrary high frequency resonance with large amplitude can lead to instabilities at a multitude of frequencies above $f_{M}$ due to feedback mechanisms. Fortunately, the inclusion of additional uncoupled filtering circuit elements is relatively inexpensive in comparison to the computationally more demanding coupled circuit elements. As an alternative approach to our filtering approach, the geometrical arrangements of the delay distances is used in [11] to improve the phase response for very high frequencies.

\section{A. Partial coefficients of potential filter}

The main issue is how the low pass filter circuits can be designed in conjunction with the subdivided partial coefficients of potential with the least number of additional circuit elements.

1) R-cap filter: This task can be accomplished by modifying the classical PEEC elementary circuit as shown in Fig. 4. The conventional modified nodal analysis (MNA) circuit implementation for PEEC includes a zero valued $V_{s}=0$ voltage sources connected between the general nodes $k$ (= $\alpha$ and $\beta)$ and $\ell\left(=\alpha^{\prime}\right.$ and $\left.\beta^{\prime}\right)$ which connects to the selfcapacitances in Fig. 4. Then the voltage source matrix stamp to measure the total current for the capacitive current is given by

\begin{tabular}{|c||c|c|c|c|}
\hline Row & $V_{k}$ & $V_{\ell}$ & $I_{s}$ & RHS \\
\hline \hline $\mathrm{k}$ & 0 & 0 & 1 & 0 \\
$\ell$ & 0 & 0 & -1 & 0 \\
$\mathrm{~m}$ & 1 & -1 & 0 & $V_{s}$ \\
\hline
\end{tabular}

Importantly, if we replace the zero voltage voltage source with a small resistor $R_{s}$, the only difference will be that the diagonal term is non-zero with almost the same stamp, or

\begin{tabular}{|c||c|c|c|c|}
\hline Row & $V_{k}$ & $V_{\ell}$ & $I_{s}$ & RHS \\
\hline \hline $\mathrm{k}$ & 0 & 0 & 1 & 0 \\
$\ell$ & 0 & 0 & -1 & 0 \\
$\mathrm{~m}$ & 1 & -1 & $-R_{s}$ & $V_{s}$ \\
\hline
\end{tabular}

Hence, this matrix stamp has the purpose of providing the capacitive current as an unknown, as well as the introduction of the very small damping resistance which is only significant in impedance in comparison to the partial $s / p_{i i}$ for frequencies above $f_{M}$. The equivalent circuit for the new PEEC circuit with the filter resistor is shown in Fig. 4. The computational cost for this $R$-cap filter is very low.

2) Split-cap filter: A second technique called Split-cap filter is proposed to eliminate unwanted resonances at high frequencies. It consists of a splitting of the partial self coefficients into two parts as shown in Fig. 5. The idea is to modify the self capacitance representation with an additional resistor filter for high frequencies $\left(f>>f_{M}\right)$, where the cutoff of this filter is again at $20 f_{M}$.

\section{B. Partial inductance (R-ind) filter}

We consider the coupled delayed sources which occur due to the delayed mutual inductance coupling voltage sources as 


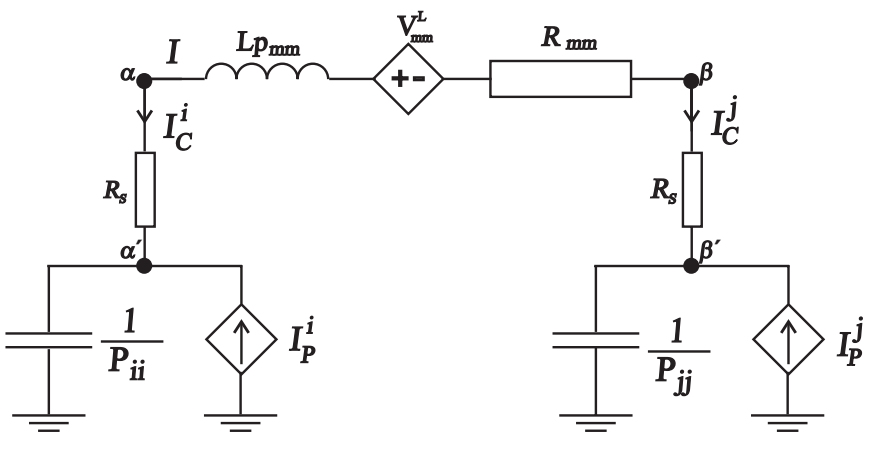

Fig. 4. The basic PEEC cell with the R-cap filter added.

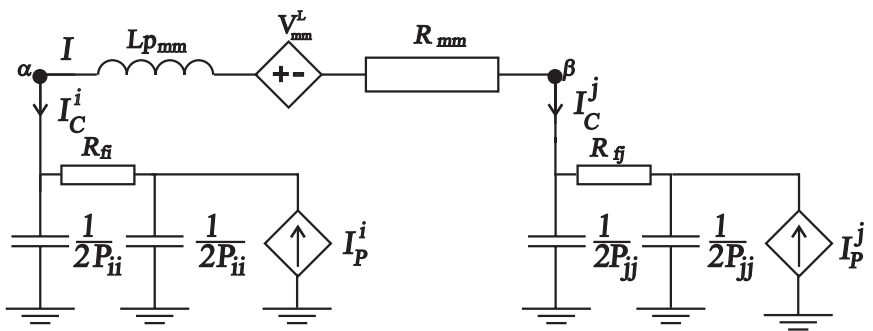

Fig. 5. The basic PEEC cell with the Split-cap filter without $R$-cap filter.

indicated in Fig. 6. A resistor is placed across each inductive branch to dampen the voltages coupled by the multitude of inductive couplings with delays at very high frequencies at $f=10 f_{M}$ to $20 f_{M}$ which does not impact the active frequency range. We observe similar resonances as for the capacitive coupling which may be responsible for instabilities. Hence, the filtering of coupled signals is as important for the partial inductances as it is for the potential coefficients. In [9] an approach was suggested for the inductive filtering where a large damping resistor was placed across each partial inductance. Not surprisingly, this was found to be only partially effective without capacitive filter in the stabilization effort. For the inductive filtering we consider the transimpedance from the current in another branch, $I_{2}$ to the coupled voltage $V_{01}$ in Fig. 6 where $V_{01}=Z_{12} I_{2}$. The damping resistance is again chosen such that the resistance is equal to the inductive impedance at 10 to $20 f_{M}$ to assure the proper functioning of the model in the extended frequency range.

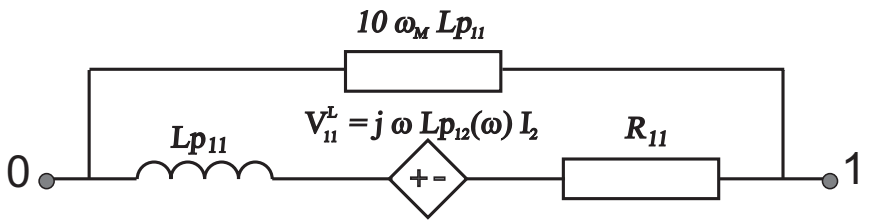

Fig. 6. Inductive branch of PEEC with $R$-ind filter.

\section{NumeriCAl Results FOR THE FD}

This section shows results for the different filter configurations detailed in the previous sections.

\section{A. Coefficients of potential}

1) Near field coupling (two touching $\frac{\lambda_{\min }}{20}$ square cells): In the first numerical example, two square cells are considered, $\alpha$ and $\beta$, with dimensions:

- $l_{\alpha}=l_{\beta}=1.5 \mathrm{~mm}$,

- $w_{\alpha}=w_{\beta}=1.5 \mathrm{~mm}$,

- $D l=1.5 \mathrm{~mm}$, and $D w=0 \mathrm{~mm}$

from Fig. 7. This ensures that the cell dimensions match the requirement $\max \left(l_{\alpha}, l_{\beta}, w_{\alpha}, w_{\beta}\right) \leq \lambda_{\min } / 20$ at the active frequency $f_{a}=10 \mathrm{GHz}$.

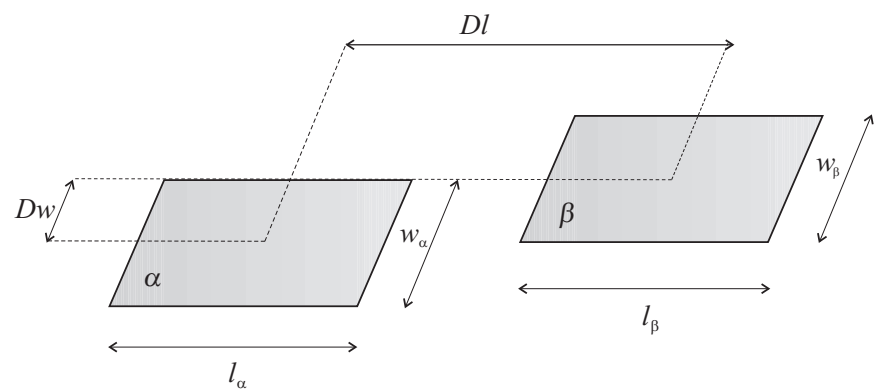

Fig. 7. Two elementary surface PEEC cells $\alpha$ and $\beta$ (rectangular cells created by structured meshing routines).

The mutual normalized impedance of the coefficient of potential is $Z_{C, \alpha \beta}^{d l}(s)=P_{\alpha \beta}(s) / s$. The results of the filtered potential coefficient by the $R$-cap filter is shown in Fig. 8. The voltage at the node $\alpha$ is given by

$$
V_{\alpha}^{C}=Z_{C, \alpha \beta} I_{C}^{\beta}
$$

where the coupling exists for all the other nodes. The $R$-cap filter introduces a voltage divider where the resistor dominates at very high frequencies $f>>f_{M}$ over the capacitive impedance reducing the coupled current.

As a second example, we compare the conventional model with the Split-cap filter detailed in Fig. 5. The test shows the node potential $\varphi_{\alpha}$ for a coupled current (injected to model the electric field coupling from surface cell $\beta$ ) with magnitude $I_{P}^{i}=\frac{p 12}{p 11}$. The results are shown in Fig. 9 and in this case it is again obvious that the resonance peaks which lead to potential instabilities are substantially reduced in magnitude. The examples for these cases are given up to $2 \mathrm{THz}$.

2) Far field coupling (two separated $\frac{\lambda_{\min }}{20}$ square cells): We also need to verify that the filtering approach applied to the far coefficients. The far couplings have smaller magnitudes but electrically large problems have a very large number of these coefficients. The dimensions of the cells in the example in Fig. 7, for $\alpha$ and $\beta$, are: 


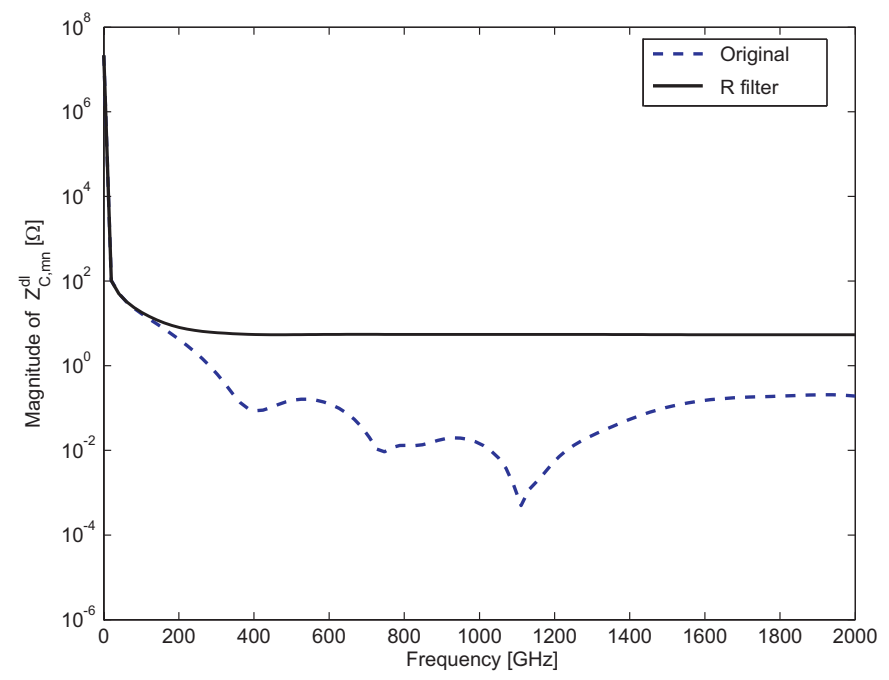

Fig. 8. Magnitude of mutual close cell impedance $Z_{C, \alpha \beta}^{d l}$ including the $R$-cap filter.

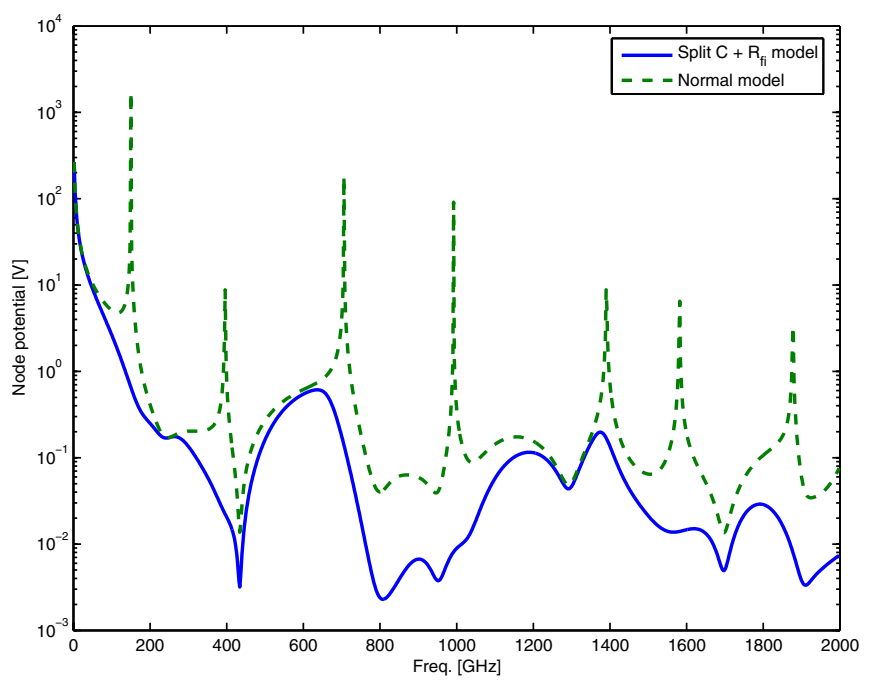

Fig. 9. Impact of Split-cap filter on the node potential for close cells.

- $l_{\alpha}=l_{\beta}=1.5 \mathrm{~mm}$,

- $w_{\alpha}=w_{\beta}=1.5 \mathrm{~mm}$,

- $D l=15 \mathrm{~mm}$, and $D w=15 \mathrm{~mm}$.

It is evident from the response in Fig. 10 that the far field coupling is similar to the closely located cells since the phase behavior dominates over the $1 / R$ distance behavior.

\section{B. Partial inductances}

Figure 11 shows the comparison of the inductive coupling for an example with $f_{A}=10 \mathrm{GHz}$ where we show the voltage coupled to the branch for a constant input current. The quasistatic coupling shown (QS in Fig. 11) is linear with frequency, as expected for an inductor while the subdivided delayed model (Subd. in Fig. 11) shows the usual resonance peaks. As we observe, they may occur at very high frequencies, for

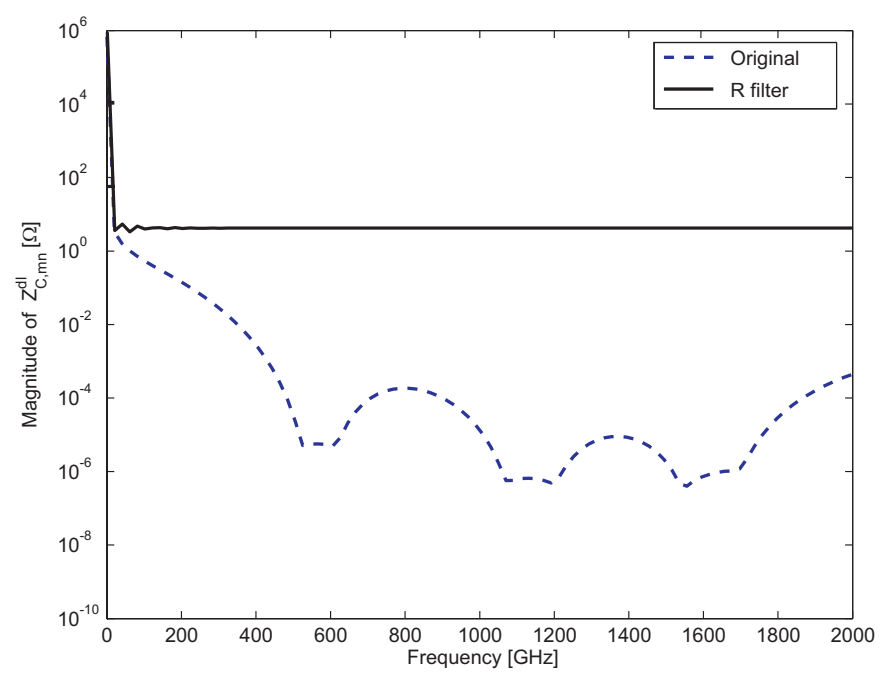

Fig. 10. Magnitude of the far mutual impedance $Z_{C, \alpha \beta}^{d l}$ for the $R$-cap filter.

this example at roughly $3.0,3.7,4.3,4.4$, and $4.8 \mathrm{THz}$. These resonances are also found in the response when using numerical integration to evaluate the frequency dependent couplings (Num.int. in Fig. 11). However, the filtered response (Subd. $+R$-ind filter in Fig. 11) shows excellent damping above $f_{M}=100 \mathrm{GHz}$. Hence, we again can keep the couplings which may cause resonance small.

\section{NUMERICAL EXPERIMENT IN THE TD}

We give an example to illustrate the stability problem. While the stability problem does not occur in the frequency domain it does manifest itself as a non-passivity issue. So, it is safe to say that the problem is related to both the time and frequency domain. The unpredictability of the stable and unstable regions was always surprising to us. However, it we observe the seemingly randomness of the very high frequency resonance peaks in the coupling coefficient, it is not surprising.

Our example consists of a $2 \mathrm{~cm}$ terminated transmission line which is excited with a pulse with 10 ps base width. We used a PEEC model with about 21000 unknowns to test a larger number of unknowns. We observe that the filtered model response is stable while the unfiltered response is unstable. Also, as a further example we chose the filter cutoff frequency such that it slightly dampens the response in the active region below $f_{A}$ for the fast input rise time.

\section{CONClusions}

The filtering approach presented in this paper eliminates very high frequency eigenvalues which are created by the discretization. These eigenvalues with large residues may lead to the very high frequency time domain instabilities. Fortunately, the circuit filters introduced in the PEEC model 


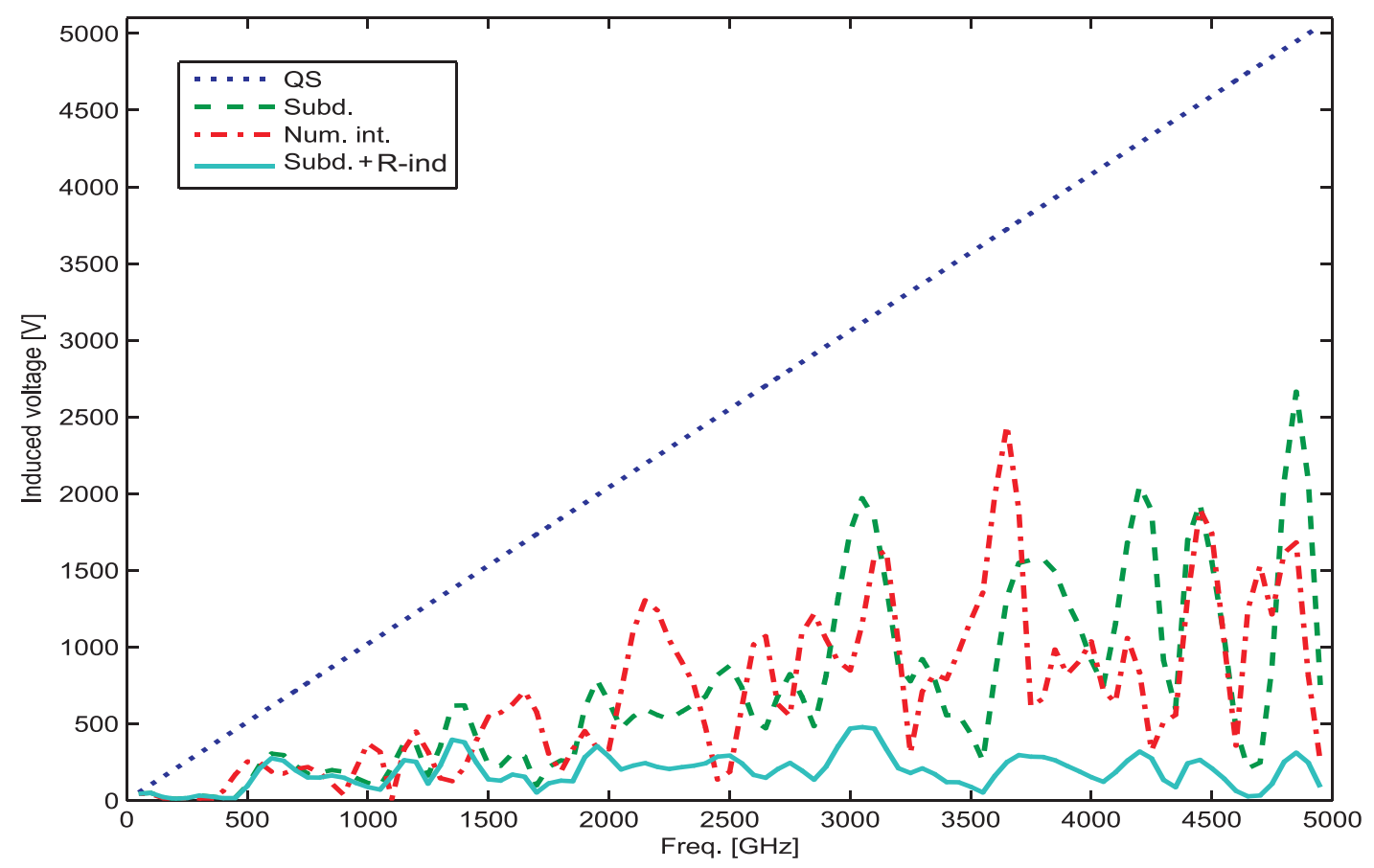

Fig. 11. Magnetic field coupling between two PEEC volume cells for different cases. Subdivided $L p_{i j}$ with damping resistor (Subd. $+\mathrm{R}$-ind) gives the desired coupling characteristic without large resonance peaks.

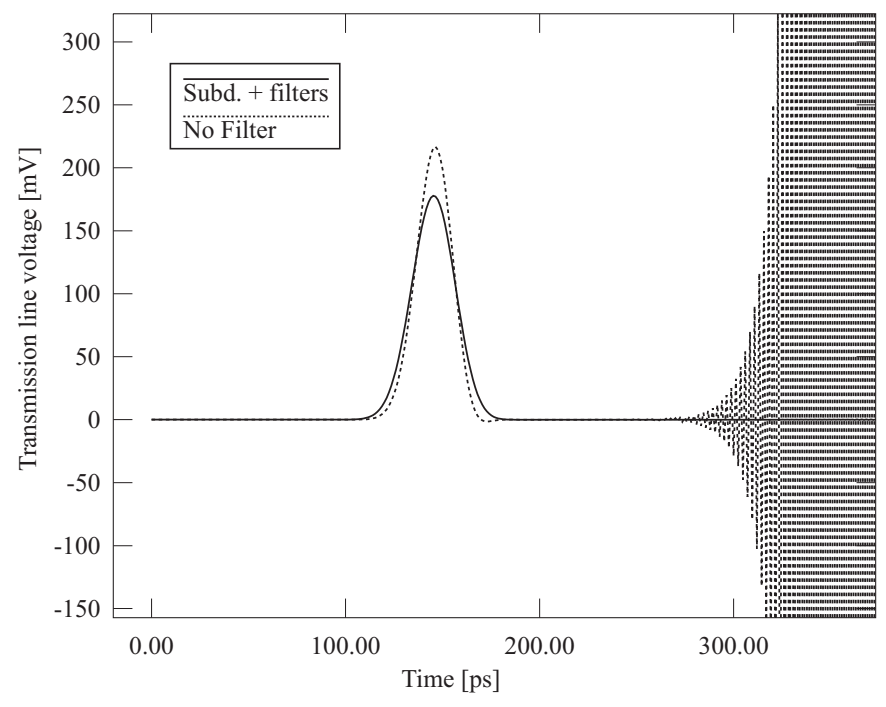

Fig. 12. Stabilization of small transmission line example using the proposed filters in combination with subdivisions.

and detailed in this paper efficiently filters the unwanted resonances. Further, the filter configurations are computationally not very costly. The resultant PEEC circuit model can be applied in the time and the frequency domain with better passivity properties.

\section{REFERENCES}

[1] A. E. Ruehli. Inductance calculations in a complex integrated circuit environment. IBM Journal of Research and Development, 16(5):470481, September 1972.

[2] A. E. Ruehli. Equivalent circuit models for three dimensional multiconductor systems. IEEE Trans. on Microwave Theory and Techniques, MTT-22(3):216-221, March 1974.

[3] B.P. Rynne. Comments on a stable procedure in calculating the transient scattering by conducting surfaces of arbitary shape. IEEE Trans. on Antennas and Propagation, APP-41(4):517-520, April 1993.

[4] G. Antonini, A. E. Ruehli, and J. Ekman. Stability of PEEC models with respect to partial element accuracy. In Proc. of the IEEE Int. Symp. on EMC, Barcelona, Spain, September 2006.

[5] A. E. Ruehli, G. Antonini, and J. Ekman. Neutral delay differential equations from the PEEC circuit solution of maxwell's equation. In Sixth IFAC Workshop on Time-Delay Systems, L'Aquila, Italy, 2006.

[6] S. V. Kochetov, G. Wollenberg. Stability of full-wave PEEC models: reason for instabilities and way for correction. In Proc. of the Int. Zurich Symposium on EMC, Zurich, SW, February 2005.

[7] S. V. Kochetov, G. Wollenberg. Stable time domain PEEC solution for pulse excited interconeection structures. In Proc. of the IEEE Int. Symp. on EMC, Chicago, USA, August 2005.

[8] G. Antonini, A. E. Ruehli. Fast Multipole and Multi-Function PEEC Methods. IEEE Transactions on Mobile Computing, 2(4), OctoberDecember 2003.

[9] J. Garrett, A.E. Ruehli, and C.R. Paul. Accuracy and stability improvements of integral equation models using the partial element equivalent circuit PEEC approach. IEEE Trans. on Antennas and Propagation, 46(12):1824-1831, December 1998.

[10] A. E. Ruehli and C. Yang. Electromagnetic response model with improved high frequency stabiblity. In United States Patent, Yor920040657US1, October 2005.

[11] J. Pingenot, S. Chakraborty, V. Jandhyala. Polar integration for exact space-time quadrature in time-domain integral equations. (submitted for publication). IEEE Trans. on Antennas and Propagation, 2006. 